\title{
Effects of single-dose atorvastatin on interleukin-6, interferon gamma, and myocardial no-reflow in a rabbit model of acute myocardial infarction and reperfusion
}

\author{
X.J. Zhao ${ }^{1 *}$, X.L. Liü ${ }^{2 *}$, G.X. He ${ }^{3}$ and H.P. Xu \\ ${ }^{1}$ Department of Cardiology, Affiliated Hospital of Binzhou Medical University, Binzhou, China \\ ${ }^{2}$ Department of Cardiology, Qilu Hospital, Shandong University, Jinan, China \\ ${ }^{3}$ Department of Cardiology, Southwest Hospital, Third Military Medical University, Chongqing, China
}

\begin{abstract}
The mechanisms of statins relieving the no-reflow phenomenon and the effects of single-dose statins on it are not well known. This study sought to investigate the effects of inflammation on the no-reflow phenomenon in a rabbit model of acute myocardial infarction and reperfusion (AMI/R) and to evaluate the effects of single-dose atorvastatin on inflammation and myocardial no-reflow. Twenty-four New Zealand white male rabbits (5-6 months old) were randomized to three groups of eight: a shamoperated group, an AMI/R group, and an atorvastatin-treated group (10 mg/kg). Animals in the latter two groups were subjected to $4 \mathrm{~h}$ of coronary occlusion followed by $2 \mathrm{~h}$ of reperfusion. Serum levels of interleukin (IL)- 6 were measured by enzyme-linked immunosorbent assay. The expression of interferon gamma (IFN- $\gamma$ ) in normal and infarcted (reflow and no-reflow) myocardial tissue was determined by immunohistochemical methods. The area of no-reflow and necrosis was evaluated pathologically. Levels of serum IL-6 were significantly lower in the atorvastatin group than in the AMI/R group $(\mathrm{P}<0.01)$. Expression of IFN- $\gamma$ in infarcted reflow and no-reflow myocardial tissue was also significantly lower in the atorvastatin group than in the AMI/R group. The mean area of no-reflow [47.01\% of ligation area (LA)] was significantly smaller in the atorvastatin group than in the AMI/R group $(85.67 \%$ of $L A ; P<0.01)$. The necrosis area was also significantly smaller in the atorvastatin group ( $85.94 \%$ of $L A)$ than in the AMI/R group $(96.56 \%$ of $L A ; P<0.01)$. In a secondary analysis, rabbits in the atorvastatin and AMI/R groups were divided into two groups based on necrosis area ( $90 \%$ of LA): a small group ( $<90 \%$ of LA) and a large group ( $>90 \%$ of LA). There was no significant difference in the area of no-reflow between the small $(61.40 \%$ of $L A)$ and large groups $(69.87 \%$ of $L A ; P>0.05)$. Single-dose atorvastatin protected against inflammation and myocardial no-reflow and reduced infarct size during AMI/R in rabbits. No-reflow was not dependent on the reduction of infarct size.
\end{abstract}

Key words: Myocardial infarction; No-reflow phenomenon; Inflammation; Atorvastatin

\section{Introduction}

The main goal of reperfusion therapy for acute myocardial infarction (AMI) is to restore epicardial and microvascular blood flow to the ischemic myocardium. The severe pathophysiological response triggered by AMI and reperfusion $(A M I / R)$ results in slow flow or no-reflow phenomena, which restore epicardial flow but provide only poor perfusion of distal tissue (1). No-reflow is a poor prognosticator for left ventricular remodeling and function, acute and long-term clinical events, and survival (2).
Therefore, reducing the extent of no-reflow has become an accepted target of reperfusion therapy for AMI (3).

The mechanism responsible for the no-reflow phenomenon is poorly understood, but it is likely to be multifactorial and involve microcirculation disturbances (4). Statins have been shown to reduce myocardial noreflow after ischemia and reperfusion $(2,5)$. However, the mechanisms involved are also poorly understood. Shortterm pretreatment with $80 \mathrm{mg}$ atorvastatin $12 \mathrm{~h}$ before

Correspondence: X.L. Liu, Department of Cardiology, Qilu Hospital, Shandong University, Jinan 250012, China. Fax: +86-021-64085875. E-mail: beyond2005388@yahoo.com.cn

${ }^{*}$ These authors contributed equally to this study.

Received April 11, 2013. Accepted October 2, 2013. First published online February 14, 2014. 
percutaneous coronary intervention $(\mathrm{PCl})$, and an additional $40 \mathrm{mg}$ preprocedure dose, has been shown to improve outcome in patients with acute coronary syndrome who undergo early, invasive intervention (6). The same investigators reported that postprocedural elevation of creatine kinase-myocardial band and troponin-I were also significantly lower in the atorvastatin-treated patients than in controls.

In the present study, we investigated the mechanisms of the no-reflow phenomenon and evaluated the effects of single-dose statins on myocardial no-reflow during AMI/R. It has been reported that no-reflow predominantly develops within the first $2 \mathrm{~h}$ of reperfusion (2). We, therefore, used a rabbit model of $4 \mathrm{~h}$ of coronary occlusion followed by $2 \mathrm{~h}$ of reperfusion to observe the effects of single-dose atorvastatin on interleukin (IL)-6, interferon gamma (IFN$\gamma)$, and myocardial no-reflow.

\section{Material and Methods}

\section{Animal model}

The animals and protocols used in the study were approved by the Institutional Animal Care and Use Committee of Binzhou Medical College.

Twenty-four New Zealand white male rabbits 5 to 6 months old and weighing 2.5 to $3.0 \mathrm{~kg}$ were anesthetized intravenously with $30 \mathrm{mg} / \mathrm{kg}$ pentobarbital sodium and ventilated with a respirator (SV900; SiemensElema, Sweden) using room air enriched with $1.5 \mathrm{~L} / \mathrm{min}$ oxygen. A left lateral thoracotomy was performed in the third to fourth intercostal space, and the heart was suspended in a pericardial cradle. The middle portion of a major branch of the left circumflex coronary artery (LCX) was encircled with a suture. The two ends of the suture were threaded through a piece of plastic tubing to form a snare that could be tightened to achieve coronary artery occlusion.

The animals were divided randomly into three groups of eight: a sham-operated group, an AMI/R group, and group that received a single $10 \mathrm{mg} / \mathrm{kg}$ dose of atorvastatin (Pfizer Pharmaceuticals Limited, USA) $12 \mathrm{~h}$ before the experiment. The $A M I / R$ and atorvastatin groups were subjected to $4 \mathrm{~h}$ of coronary occlusion followed by $2 \mathrm{~h}$ of reperfusion. In the sham-operated animals, the LCX was encircled by a suture, but not occluded. Data were collected at baseline, at the end of $4 \mathrm{~h}$ of LCX occlusion, and after $2 \mathrm{~h}$ of reperfusion. All procedures were carried out as previously described $(5,7)$.

\section{Experimental protocol}

After completion of the experimental procedure, the area of no-reflow (ANR) was delineated by intra-atrial injection of $1 \mathrm{~mL} / \mathrm{kg}$ of the fluorescent dye thioflavin $\mathrm{S}$ (Sigma Chemical Co., USA) that had been dissolved in $0.9 \%$ saline and then centrifuged at $1500 \mathrm{rpm}$ for $5 \mathrm{~min}$. The LCX was then re-occluded, and Evans blue dye was injected into the left atrium to determine the ligation area (LA). The animals were then euthanized by an overdose of xylazine $(100 \mathrm{mg}, i \mathrm{v})$ and $12 \mathrm{mEq} \mathrm{KCl}$ (intra-atrial), and the hearts were removed. The left ventricle was cut into five or six slices parallel to the atrioventricular groove. Areas not perfused by thioflavin $S$ were identified under ultraviolet light in a dark room. The LA was defined as the region unstained by Evans blue dye, and the ANR was defined as the nonfluorescent area within the LA.

The other slices were incubated in a $1 \%$ solution of triphenyltetrazolium chloride for $15 \mathrm{~min}$ at $37^{\circ} \mathrm{C}$. Regions that failed to demonstrate red staining were considered to represent areas of necrosis (NA). All slices were photographed. The outline of the left ventricular wall area, LA, ANR, and NA were analyzed using the Image-Pro Plus software (Media Cybernetics Co., USA). LA is reported as a percentage of the left ventricular wall area; ANR and NA are reported as a percentage of the $L A$.

After ANR and NA were evaluated pathologically, the rabbits in the atorvastatin and $A M I / R$ groups were each divided into two groups by the size of the NA, i.e., a small group and a large group, to analyze whether no-reflow was dependent on the reduction of infarct size.

\section{Measurement of serum IL-6}

Two-milliliter blood samples were collected 5 min before LCX occlusion, at the end of $4 \mathrm{~h}$ of LCX occlusion, and after $2 \mathrm{~h}$ of reperfusion. The serum was separated from blood cells by centrifugation at $2000 \mathrm{~g}$ for $10 \mathrm{~min}$ at $4^{\circ} \mathrm{C}$ and stored at $-20^{\circ} \mathrm{C}$. Serum IL-6 levels were determined using an enzyme-linked immunosorbent assay (Xi-Tang Biotechnology Company, China) and a microplate reader (Anthos Company, Austria).

\section{Assay of IFN- $\gamma$ in myocardial tissue}

Myocardial tissue samples were obtained from the normal, reflow, or no-reflow tissue slices immediately after the experimental procedures. The samples were washed in $0.9 \%$ saline, fixed in Paraform (40 g/L), embedded in paraffin, and microtome sections were obtained. Expression of IFN- $\gamma$ was assayed using an immunohistochemical technique. The sections were observed under a light microscope $(200 \times)$ and photographed. Three sections were randomly selected from each group, and three visual fields were randomly selected from each section for semiquantitative analysis using the Image-Pro Plus software (Media Cybernetics Co.). IFN- $\gamma$ positivity is reported as the average integral optical density of the nine visual fields evaluated.

\section{Statistical analysis}

Statistical analysis was performed using SPSS version 13.0 for Windows (SPSS Inc., USA). Data are reported as means $\pm S D$. IFN- $\gamma$ level, $L A, A N R$, and NA were compared among groups by one-way ANOVA 

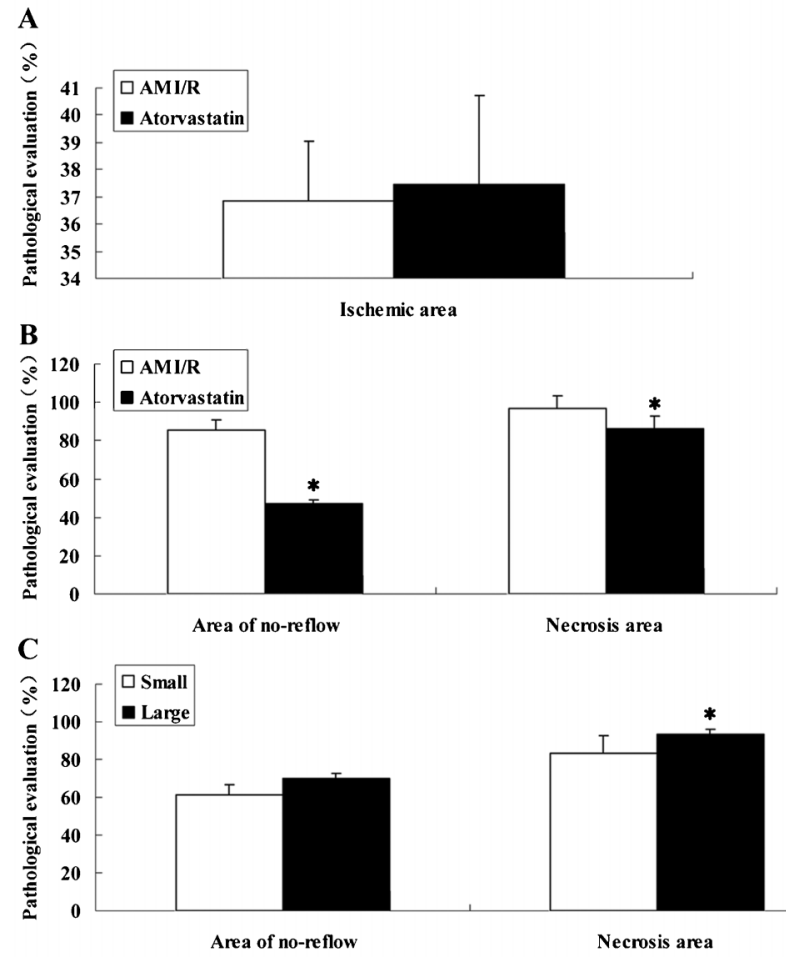

Figure 1. $A$, Comparison of ischemic area (ligation area) between two groups. $B$, Comparison of area of no-reflow and necrosis area between two groups. C, Comparison of area of no-reflow and necrosis area between small and large groups. AMI/R: acute myocardial infarction and reperfusion. ${ }^{*} \mathrm{P}<0.01$, compared to $\mathrm{AMI} / \mathrm{R}$ or small groups (one-way ANOVA followed by the Student-Newman-Keuls test and the Student $t$-test).

followed by the Student-Newman-Keuls test for multiple comparisons. ANR and NA between the small and large groups were analyzed using the Student $t$-test. IL- 6 data were compared by repeated measures ANOVA followed by the Student-Newman-Keuls test for multiple comparisons. Two-sided values of $\mathrm{P}<0.05$ were considered to be statistically significant.

\section{Results}

\section{Atorvastatin was associated with a decreased ANR and NA}

There was no significant difference in the pathological evaluation of LA between the atorvastatin $(37.45 \pm 3.25 \%)$ and $\mathrm{AMI} / \mathrm{R}(36.87 \pm 2.16 \% ; \mathrm{P}>0.05)$ groups. The mean ANR was significantly smaller in the atorvastatin group $(47.01 \pm 6.89 \%$ of $L A)$ than in the $A M I / R$ group $(85.67 \pm 4.94 \%$ of $L A ; P<0.01)$. The $N A$ in the atorvastatin group (85.94 $\pm 7.01 \%$ of LA) was also significantly smaller than in the $A M I / R$ group $(96.56 \pm 2.26 \%$ of $L A ; P<0.01)$ (Figure $1 \mathrm{~A}$ and $\mathrm{B}$, Figure 2). No-reflow was not dependent on the reduction of infarct size.
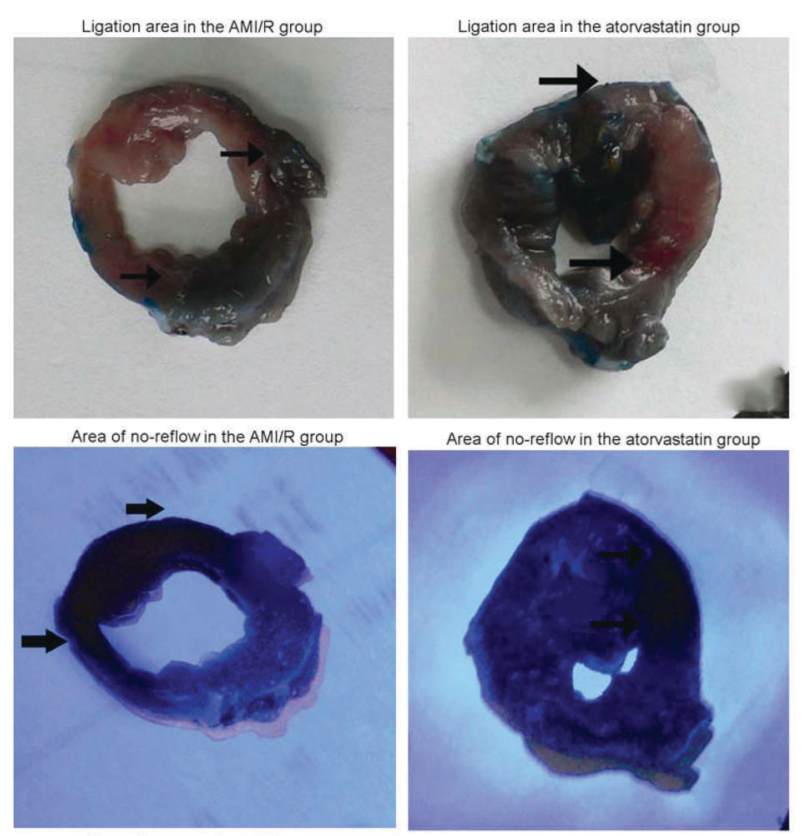

Necrosis area in the AMI/R group

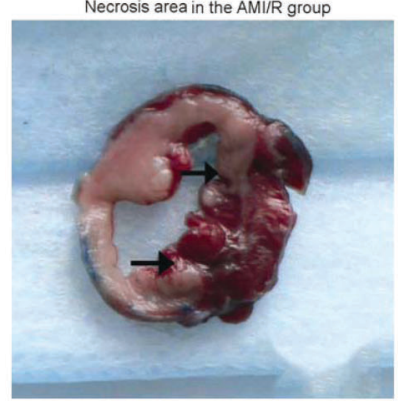

Necrosis area in the atorvastatin group

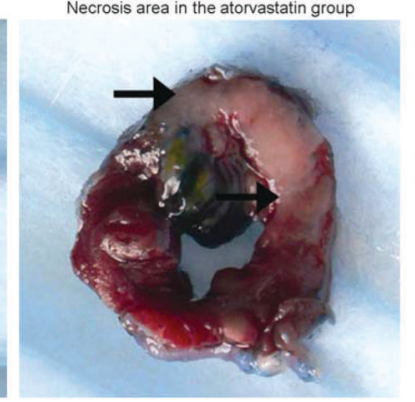

Figure 2. Ligation area, area of no-reflow and necrosis area after $4 \mathrm{~h}$ of occlusion and $2 \mathrm{~h}$ of reperfusion (pathological staining, $1 \times)$. The red areas show the ligation area, black areas show the area of no-reflow, and white areas show necrosis (see black arrows). AMI/R: acute myocardial infarction and reperfusion.

In a secondary analysis, rabbits in the atorvastatin and $\mathrm{AMI} / \mathrm{R}$ groups were divided into two groups based on the NA $(90 \%$ of LA). The small group ( $<90 \%$ of LA) included 6 rabbits, and the large group ( $>90 \%$ of LA) included 10 rabbits. There was a significant difference in pathological evaluation of the NA between the small $(83.23 \pm 3.11 \%)$ and large groups $(93.46 \pm 2.65 \% ; \mathrm{P}<0.01)$. There was no significant difference in the ANR between the small $(61.40 \pm 5.13 \%)$ and large groups $(69.87 \pm 9.16 \%$; $\mathrm{P}>0.05$ ) (Figure 1C).

Atorvastatin was associated with reduced levels of IL-6

Serum IL-6 levels did not differ significantly at any time point in the sham-operated group, and no significant differences were seen between groups at $5 \mathrm{~min}$ before LCX occlusion. In the AMI/R and atorvastatin groups, serum IL-6 significantly increased from baseline, after $4 \mathrm{~h}$ 


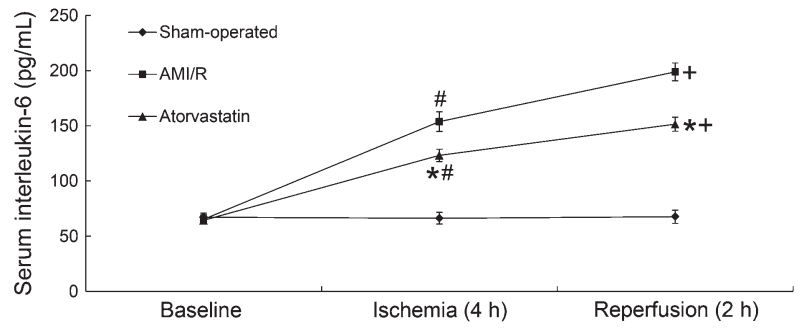

Figure 3. Effects of atorvastatin on serum interleukin-6 at different time points. ${ }^{*} \mathrm{P}<0.01$, compared to acute myocardial infarction and reperfusion $(\mathrm{AMI} / \mathrm{R}) ;{ }^{\#} \mathrm{P}<0.01$, compared to baseline; ${ }^{+} \mathrm{P}<0.01$, compared to ischemia (4 h) (repeated measures ANOVA followed by the Student-Newman-Keuls test).

of LCX occlusion and after $2 \mathrm{~h}$ of reperfusion (all $\mathrm{P}<0.01$ ). However, IL-6 levels in the atorvastatin group were significantly lower than in the AMI/R group (all $P<0.01$; Figure 3).

\section{Atorvastatin was associated with reduced expression of IFN- $\gamma$ in myocardial tissue subjected to reflow and no-reflow}

Immunohistochemistry clearly demonstrated positive

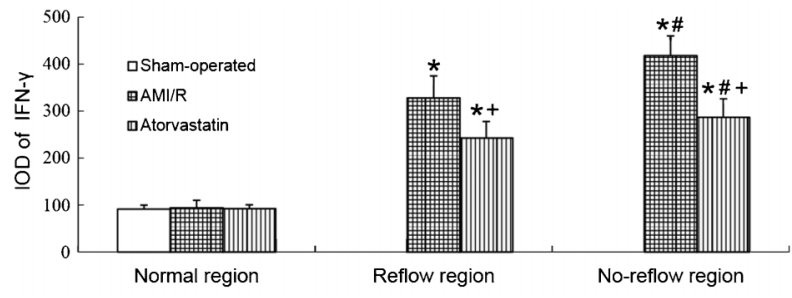

Figure 5. Integral optical density (IOD) analysis of IFN- $\gamma$ levels in myocardium of different regions in three groups. ${ }^{*} \mathrm{P}<0.01$, compared to normal region; ${ }^{\#} \mathrm{P}<0.01$, compared to reflow region; ${ }^{+} \mathrm{P}<0.01$, compared to acute myocardial infarction and reperfusion (AMI/R) (one-way ANOVA followed by the StudentNewman-Keuls test).

expression of IFN- $\gamma$ in vascular endothelial cells (Figure 4). As shown in Figure 5, IFN- $\gamma$ expression during reflow and no-reflow was significantly higher in the AMI/R and atorvastatin groups than in normal myocardium or in the sham-operated group (all $\mathrm{P}<0.01$ ). In both groups, IFN- $\gamma$ expression was more marked in no-reflow areas than in reflow areas of the myocardium (both $\mathrm{P}<0.01$ ). However, IFN- $\gamma$ expression in the reflow and no-reflow myocardial areas was lower in the atorvastatin group than in the AMI/
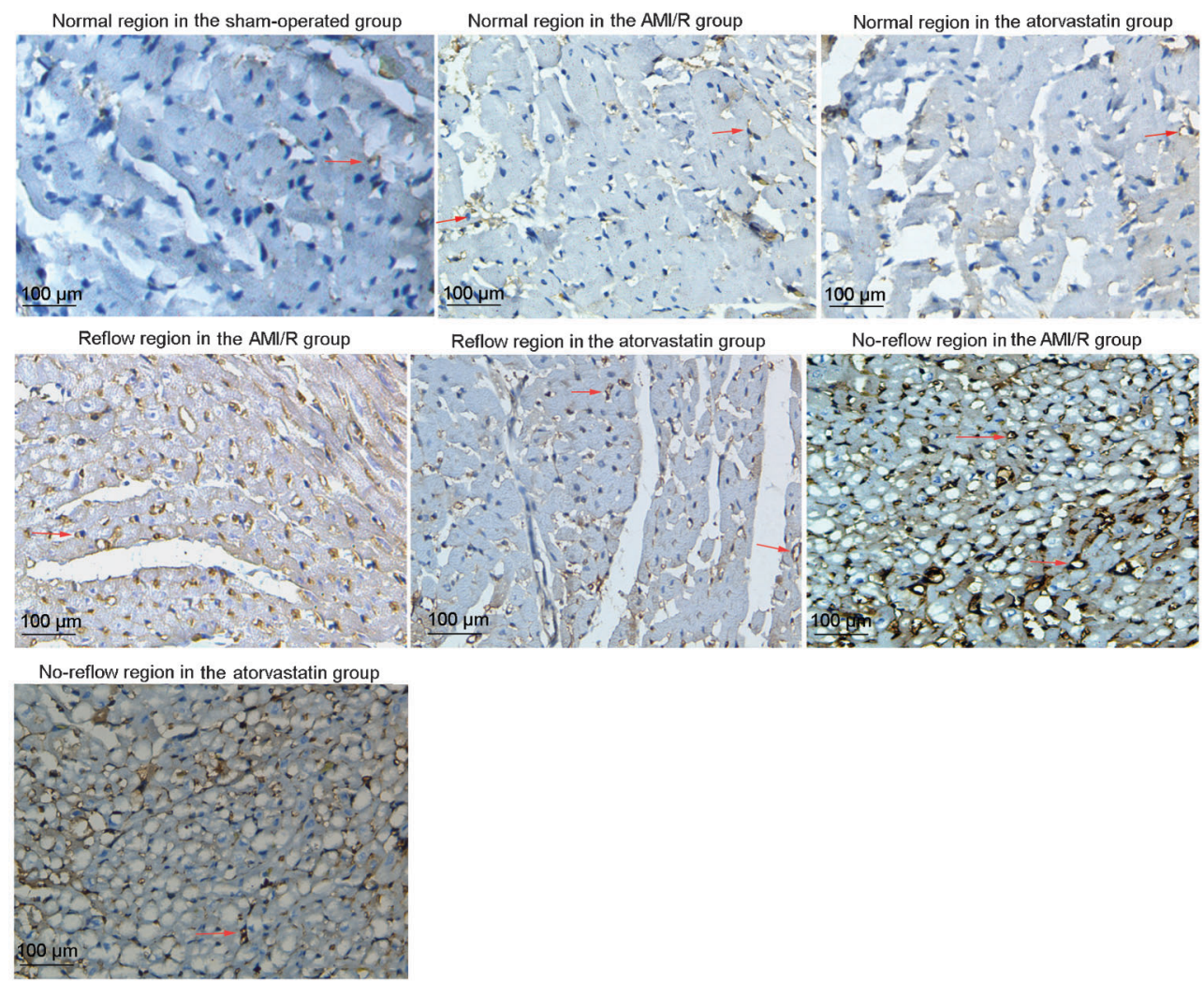

Figure 4. Expression of IFN- $\gamma$ in different regions of the myocardium in the three groups after $4 \mathrm{~h}$ of occlusion and $2 \mathrm{~h}$ of reperfusion (IHC, DAB staining, $200 \times$ ). Positive IFN- $\gamma$ expression was seen in vascular endothelial cells (as shown by arrows). AMI/R: acute myocardial infarction and reperfusion. 
$\mathrm{R}$ group (both $\mathrm{P}<0.01$ ).

\section{Discussion}

Statins are widely used to treat dyslipidemias. Cardioprotection elicited by this class of drugs may be attributed to their diverse variety of non-lipid lowering pleiotropic effects. Statins augment endothelial function, stabilize vulnerable plaque, reduce adhesion molecules, and decrease circulating biomarkers $(8,9)$. It has become increasingly clear that the cardioprotective actions of statins are not limited to the prevention of cardiovascular disease, but include acute effects that have been shown to be mediated by mechanisms independent of cholesterol lowering (10).

Statins have been shown to provide protection against ischemia-reperfusion injury (11). The Novel Approaches for Preventing or Limiting Events (NAPLES II) study showed that a single, loading dose of statin was associated with a $32 \%$ reduction in the incidence of peri-procedural biomarker-defined myocardial infarction (MI) in patients who underwent elective $\mathrm{PCl}$ (12). This result supported the findings of an earlier study (13). However, there is a paucity of data reporting the effects of single-dose statins on myocardial tissue-level perfusion when given $12 \mathrm{~h}$ before ischemia and reperfusion. This has prevented statins from being routinely used in cardiac emergencies.

Our results show that single-dose atorvastatin significantly reduced the mean area of no-reflow following $\mathrm{AMI} / \mathrm{R}$. Chronic statin treatment before admission has been shown to reduce the incidence of no-reflow in patients with acute $\mathrm{MI}$ undergoing successful primary $\mathrm{PCI}$ (14). Other investigators have shown that pretreatment of animals with simvastatin before reperfusion significantly decreased the plasma activity of creatine kinase, which is an index of myocardial necrosis, and also reduced both no-reflow and infarct size (15). The results of this study demonstrated that the NA in the atorvastatin group was significantly smaller than that in the AMI/R group. This finding suggests that atorvastatin may have a protective effect on the myocardium as proposed by others $(15,16)$. It has previously been demonstrated that infarct size may be associated with the no-reflow phenomena (17).

IL-6 is an important mediator of inflammation, which is generated by leukocyte and endothelial cells. It has been suggested that IL- 6 is more sensitive than C-reactive protein or creatine kinase during myocardial ischemia and reperfusion (18), and, on the basis of these findings, it is regarded as an important marker of myocardial tissue stress and injury. Our results indicated that both ischemia and reperfusion injury induced the production and release of serum IL-6. We also showed that a single dose of atorvastatin was able to decrease IL-6 levels, possibly resulting in an anti-inflammatory effect. Experimental studies have shown that pretreatment with simvastatin reduces systemic and myocardial levels of proinflammatory cytokines by stimulating peroxisome proliferatoractivated receptor gamma receptors and by inhibiting nuclear factor- $\kappa B$ expression in myocardial tissue (19). In addition to suppressing the expression of proinflammatory cytokines, statins have also been shown to upregulate the expression of the anti-inflammatory cytokine IL-10 and to improve the ratio of tumor necrosis factor (TNF)- $\alpha / \mathrm{IL}-10$ in a post-MI model in rats (20). These effects may ameliorate the early phases of left ventricular remodeling that occur post-MI and thereby improve left ventricular function (20).

IFN- $\gamma$ is an inflammatory cytokine secreted by endothelial cells, mononuclear macrophages, and lymphocytes. It participates in immunopathogenesis and destroys the structure and function of blood vessel endothelium $(21,22)$. It has also been shown that IFN- $\gamma$ increases excretion of C-reactive protein, which accelerates the development of atherosclerosis and thrombosis (23). Our findings indicated that both ischemia and reperfusion injury can induce inflammatory responses that promote IFN- $\gamma$ production and release in the myocardium. We also showed that atorvastatin inhibits IFN- $\gamma$ expression in the myocardium and thereby protects against inflammation. Statins have previously been shown to modulate the activity of transcriptional factors in myocardial tissue. Atorvastatin was reported to improve survival in a murine model of viral myocarditis by decreasing myocardial TNF- $\alpha$ and IFN- $\gamma$ expression and increasing the expression of connexins (24). In a study in patients with troponin-positive acute coronary syndrome, rosuvastatin significantly reduced plasma concentrations of IFN- $\gamma$ and induced a rapid and significant reduction of IFN- $\gamma$ production in stimulated T-lymphocytes (25).

The mechanism of microvascular dysfunction leading to no-reflow is complex. Microvascular spasm, distal embolization of atheroma and thrombus, microvascular plugging by platelets and leukocytes, endothelial swelling, tissue edema resulting in compression of the microvasculature, oxidative stress, and inflammation may all play a role (26). Using a rabbit model of coronary occlusion and reperfusion, we demonstrated that $\mathrm{AMI}$ and reperfusion resulted in a significant increase in serum IL-6 levels and IFN- $\gamma$ expression in myocardial tissue. We also showed that IFN- $\gamma$ expression was most marked in no-reflow regions of the myocardium. It has been demonstrated that severe inflammatory reactions occurring during AMI/R can result in coronary microcirculation dysfunction and induce no-reflow (27). Thus, inflammation may be an important mechanism that contributes to no-reflow.

A secondary analysis in this study revealed a significant difference in NA between the small and large groups (i.e., the extent relative to the LA), but there was no significant difference in ANR between the small and large groups. This showed that no-reflow was not dependent on the reduction of infarct size. This result is 
in agreement with the findings of an earlier study (28). Microvascular obstruction is a cardiac magnetic resonance marker of no-reflow in ST-segment elevation myocardial infarction (STEMI). The presence of microvascular obstruction but not its extent corresponds to a larger infarct size in STEMI.

We clearly demonstrated that single-dose atorvastatin induced a reduction in serum IL-6, and myocardial IFN- $\gamma$ expression resulted in a reduction in the ANR and infarct size during $\mathrm{AMI}$ and reperfusion. The means by which atorvastatin reduced infarct size is not clear. Several mechanisms have been proposed including prevention of myocardial no-reflow (17), anti-inflammatory effects $(10,29,30)$, preservation of endothelial function (31), modulation of nitric oxide synthetase expression (32), protection from ischemia-reperfusion injury (11), increased coronary blood flow (33), and inhibition of platelet aggregation and thrombus formation (34).

A significant body of clinical research and experimental evidence obtained in the past decade has established important prognostic implications of the occurrence and extent of no-reflow for recovery of

\section{References}

1. Gibson CM, Cannon CP, Murphy SA, Ryan KA, Mesley R, Marble SJ, et al. Relationship of TIMI myocardial perfusion grade to mortality after administration of thrombolytic drugs. Circulation 2000; 101: 125-130, doi: 10.1161/01.CIR. 101.2.125

2. Schwartz BG, Kloner RA. Coronary no reflow. J Mol Cell Cardiol 2012; 52: 873-882, doi: 10.1016/j.yjmcc.2011.06. 009.

3. Gersh BJ. Optimal management of acute myocardial infarction at the dawn of the next millennium. Am Heart $J$ 1999; 138: S188-S202, doi: 10.1016/S0002-8703(99) 70342-X.

4. Kloner RA. No-reflow phenomenon: maintaining vascular integrity. J Cardiovasc Pharmacol Ther 2011; 16: 244-250, doi: $10.1177 / 1074248411405990$

5. Zhao JL, Yang YJ, Cui CJ, You SJ, Gao RL. Pretreatment with simvastatin reduces myocardial no-reflow by opening mitochondrial K(ATP) channel. Br J Pharmacol 2006; 149: 243-249, doi: 10.1038/sj.bjp.0706862.

6. Patti G, Pasceri V, Colonna G, Miglionico M, Fischetti D, Sardella G, et al. Atorvastatin pretreatment improves outcomes in patients with acute coronary syndromes undergoing early percutaneous coronary intervention: results of the ARMYDA-ACS randomized trial. J Am Coll Cardiol 2007; 49: 1272-1278, doi: 10.1016/j.jacc.2007.02.025.

7. Reffelmann T, Kloner RA. Microvascular reperfusion injury: rapid expansion of anatomic no reflow during reperfusion in the rabbit. Am J Physiol Heart Circ Physiol 2002; 283: H1099-H1107.

8. Wang CY, Liu PY, Liao JK. Pleiotropic effects of statin therapy: molecular mechanisms and clinical results. Trends Mol Med 2008; 14: 37-44, doi: 10.1016/j.molmed.2007.11. 004. regional myocardial function and clinical outcome. There is also evidence that statins have a favorable impact on clinical outcome. Based on our findings, we believe that the beneficial effects of statins on clinical outcomes are partly due to the reduction of myocardial no-reflow.

The present study indicates that inflammatory reactions may be associated with the no-reflow phenomenon. The anti-inflammatory effects of single-dose atorvastatin improved microcirculatory disturbance, protected against no-reflow, and reduced infarct size. In future studies, we plan to investigate whether short-term pretreatment with single-dose statins, $12 \mathrm{~h}$ before $\mathrm{PCl}$, can prevent myocardial no-reflow and improve outcomes in patients with AMI undergoing early invasive interventions.

\section{Acknowledgments}

Research supported by grants-in-aid from a project of Shandong Province Higher Educational Science and Technology Program (\#J08LH51) and from the Scientific Research Foundation for Doctors, Binzhou Medical University (\#BY2007KYQD01).
9. Nohria A, Prsic A, Liu PY, Okamoto R, Creager MA, Selwyn $A$, et al. Statins inhibit Rho kinase activity in patients with atherosclerosis. Atherosclerosis 2009; 205: 517-521, doi: 10.1016/j.atherosclerosis.2008.12.023.

10. Lefer DJ. Statins as potent antiinflammatory drugs. Circulation 2002; 106: 2041-2042, doi: 10.1161/01.CIR 0000033635.42612 .88 .

11. Meijer P, Oyen WJ, Dekker D, van den Broek PH, Wouters $\mathrm{CW}$, Boerman OC, et al. Rosuvastatin increases extracellular adenosine formation in humans in vivo: a new perspective on cardiovascular protection. Arterioscler Thromb Vasc Biol 2009; 29: 963-968, doi: 10.1161/ ATVBAHA.108.179622.

12. Briguori C, Visconti G, Focaccio A, Golia B, Chieffo A, Castelli A, et al. Novel approaches for preventing or limiting events (Naples) II trial: impact of a single high loading dose of atorvastatin on periprocedural myocardial infarction. J Am Coll Cardiol 2009; 54: 2157-2163, doi: 10.1016/j.jacc. 2009.07.005

13. Briguori C, Colombo A, Airoldi F, Violante A, Focaccio A, Balestrieri $P$, et al. Statin administration before percutaneous coronary intervention: impact on periprocedural myocardial infarction. Eur Heart J 2004; 25: 1822-1828, doi: 10.1016/j.ehj.2004.07.017

14. Iwakura K, Ito H, Kawano S, Okamura A, Kurotobi T, Date $\mathrm{M}$, et al. Chronic pre-treatment of statins is associated with the reduction of the no-reflow phenomenon in the patients with reperfused acute myocardial infarction. Eur Heart $J$ 2006; 27: 534-539, doi: 10.1093/eurheartj/ehi715.

15. Li XD, Yang YJ, Geng YJ, Zhao JL, Zhang HT, Cheng YT, et al. Phosphorylation of endothelial NOS contributes to simvastatin protection against myocardial no-reflow and infarction in reperfused swine hearts: partially via the PKA 
signaling pathway. Acta Pharmacol Sin 2012; 33: 879-887, doi: 10.1038/aps.2012.27.

16. Wolfrum S, Dendorfer A, Schutt M, Weidtmann B, Heep A, Tempel K, et al. Simvastatin acutely reduces myocardial reperfusion injury in vivo by activating the phosphatidylinositide 3-kinase/Akt pathway. J Cardiovasc Pharmacol 2004; 44: 348-355, doi: 10.1097/01.fjc.0000137162.14735.30.

17. Ferreira R. The reduction of infarct size - forty years of research. Rev Port Cardiol 2010; 29: 1037-1053.

18. Helmy SA, Wahby MA, El-Nawaway M. The effect of anaesthesia and surgery on plasma cytokine production. Anaesthesia 1999; 54: 733-738, doi: 10.1046/j.13652044.1999.00947.x.

19. Shen $Y$, Wu $H$, Wang $C$, Shao $H$, Huang $H$, Jing $H$, et al. Simvastatin attenuates cardiopulmonary bypass-induced myocardial inflammatory injury in rats by activating peroxisome proliferator-activated receptor gamma. Eur $J$ Pharmacol 2010; 649: 255-262, doi: 10.1016/j.ejphar.2010. 08.058.

20. Stumpf C, Petzi S, Seybold K, Wasmeier G, Arnold M, Raaz $D$, et al. Atorvastatin enhances interleukin-10 levels and improves cardiac function in rats after acute myocardial infarction. Clin Sci 2009; 116: 45-52, doi: 10.1042/CS200 80042.

21. Kostis JB, Turkevich D, Sharp J. Association between leukocyte count and the presence and extent of coronary atherosclerosis as determined by coronary arteriography. Am J Cardiol 1984; 53: 997-999, doi: 10.1016/0002-9149 (84)90624-6.

22. Neri Serneri GG, Boddi M, Modesti PA, Coppo M, Cecioni I, Toscano $\mathrm{T}$, et al. Cardiac angiotensin II participates in coronary microvessel inflammation of unstable angina and strengthens the immunomediated component. Circ Res 2004; 94: 1630-1637, doi: 10.1161/01.RES.0000130944. 49657.b8.

23. Yeh ET, Anderson HV, Pasceri V, Willerson JT. C-reactive protein: linking inflammation to cardiovascular complications. Circulation 2001; 104: 974-975, doi: 10.1161/01.CIR. 104.9.974.

24. Zhang A, Zhang H, Wu S. Immunomodulation by atorvastatin upregulates expression of gap junction proteins in coxsackievirus B3 (CVB3)-induced myocarditis. Inflamm Res 2010; 59: 255-262, doi: 10.1007/s00011-009-0093-8.
25. Link A, Ayadhi T, Bohm M, Nickenig G. Rapid immunomodulation by rosuvastatin in patients with acute coronary syndrome. Eur Heart J 2006; 27: 2945-2955, doi: 10.1093/ eurheartj/ehl277.

26. Herrmann J. Peri-procedural myocardial injury: 2005 update. Eur Heart J 2005; 26: 2493-2519, doi: 10.1093/ eurheartj/ehi455.

27. Maksimenko AV, Turashev AD. No-reflow phenomenon and endothelial glycocalyx of microcirculation. Biochem Res Int 2012; 2012: 859231, doi: 10.1155/2012/859231.

28. Malek LA, Spiewak M, Klopotowski M, Misko J, Ruzyllo W, Witkowski A. The size does not matter - the presence of microvascular obstruction but not its extent corresponds to larger infarct size in reperfused STEMI. Eur J Radiol 2012; 81: 2839-2843, doi: 10.1016/j.ejrad.2011.11.053.

29. Gurgun C, Ildizli M, Yavuzgil O, Sin A, Apaydin A, Cinar C, et al. The effects of short term statin treatment on left ventricular function and inflammatory markers in patients with chronic heart failure. Int J Cardiol 2008; 123: 102-107, doi: 10.1016/j.ijcard.2006.11.152.

30. Kinlay S, Schwartz GG, Olsson AG, Rifai N, Leslie SJ, Sasiela WJ, et al. High-dose atorvastatin enhances the decline in inflammatory markers in patients with acute coronary syndromes in the MIRACL study. Circulation 2003; 108: 1560-1566, doi: 10.1161/01.CIR.0000091404. 09558.AF.

31. Sakabe K, Fukuda N, Wakayama K, Nada T, Shinohara H, Tamura Y. Lipid-altering changes and pleiotropic effects of atorvastatin in patients with hypercholesterolemia. Am J Cardiol 2004; 94: 497-500, doi: 10.1016/j.amjcard.2004. 04.067.

32. Feron O, Dessy C, Desager JP, Balligand JL. Hydroxymethylglutaryl-coenzyme A reductase inhibition promotes endothelial nitric oxide synthase activation through a decrease in caveolin abundance. Circulation 2001; 103: 113-118, doi: 10.1161/01.CIR.103.1.113.

33. Lardizabal JA, Deedwania PC. The anti-ischemic and antianginal properties of statins. Curr Atheroscler Rep 2011; 13: 43-50, doi: 10.1007/s11883-010-0147-y.

34. Gaddam V, Li DY, Mehta JL. Anti-thrombotic effects of atorvastatin - an effect unrelated to lipid lowering. J Cardiovasc Pharmacol Ther 2002; 7: 247-253, doi: 10. $1177 / 107424840200700408$. 